\title{
SOME PROBLEMS OF NONLINEAR INTEGRAL EQUATIONS IN MEASURE SPACES
}

\author{
R. R. SHARMA
}

ABSTRACT. An existence theorem is proved by the method of successive approximations for abstract nonlinear integral equations formulated in [1]. Some integral inequalities and extremal solutions are also considered.

1. Introduction. Let $(X, \Re, \mu)$ be a measure space and let $\Lambda$ denote the set of all real or complex measures on $\pi$. Consider the equation

$$
\lambda(E)=\nu(E)+\int_{E-E_{0}} f\left(x, \lambda_{x}\right) d \mu
$$

where $\nu \in \Lambda, \lambda_{x}: \Lambda \times\{x\} \rightarrow R$ for each $x \in X, f: X \times R \rightarrow R, E_{0} \in \mathbb{M}$. Abstract integral equations of this form have been considered in [1]. A measure $\vec{\lambda} \in \Lambda$ is defined to be a solution of (1.1) on $\bar{E}$ if $E_{0} \subset \bar{E}$ and the equation (1.1) is satisfied for all $E \in \bar{E} \cap$ M. Note that $\bar{\lambda}$ may be considered to be a member of $\Lambda(\bar{E})$ where $\Lambda(\bar{E})$ denotes the set of restrictions of all $\lambda \in \Lambda$ to $\bar{E} \cap \mathbb{M}$. It is clear that $\bar{\lambda}$ satisfies (1.1) for all $E \in \bar{E} \cap \mathbb{M}$ if it satisfies (1.1) for $E \in \mathcal{Y}$ where $\mathcal{Y}$ is a subclass of $\bar{E} \cap \pi$ which generates the $\sigma$-algebra $\bar{E} \cap \mathbb{M}$ and is such that the restriction of every measure on $\bar{E} \cap M$ to $\mathcal{G}$ has a unique $\sigma$-additive extension to $\bar{E} \cap \Re$. Thus, for $\bar{\lambda}$ to be a solution of $(1.1)$ on $\bar{E}$, we need verify $(1.1)$ in $\biguplus$ only. $\zeta$ is called a " $g$ class" for the measurable space $(\bar{E}, \bar{E} \cap \mathbb{M})$. In [1], a theorem concerning local existence and uniqueness of solutions of (1.1) is proved, and it is shown that (1.1) includes as particular cases certain classes of integral equations and also abstract measure differential equations considered in [2], [3] and [4].

In this paper we consider the problems of local existence of solutions by successive approximations, integral inequalities and the existence of extremal solutions.

2. Existence of solutions. For $A, B \in \mathbb{M}$, we define an inclusion relation with respect to $\mu$ as follows:

Received by the editors June 13, 1974.

AMS (MOS) subject classifications (1970). Primary 34A10, 45G99, 45 N05. 
$A \subseteq B[\mu]$ if and only if $|\mu|(A-B)=0$,

where $|\mu|$ denotes the total variation measure of $\mu . A$ and $B$ are defined to be equal with respect to $\mu$ if $A \subseteq B[\mu]$ and $B \subseteq A[\mu]$. Thus, $A=B[\mu]$ if and only if $|\mu|(A \triangle B)=0$.

Let $\prod_{0}$ be the set of all elements $E \in \mathbb{M}$ for which $|\mu|(E)<\infty$. For $A$, $B \in \mathbb{M}_{0}$, define

$$
\rho(A, B)=|\mu|(A \Delta B) .
$$

It can be easily verified that $\pi_{0}$ is a metric space.

Lemma 1. Let $E \in \mathbb{M}_{0}$. If $\nu \in \Lambda(E)$ and $\nu \ll|\mu|$ on $E \cap \mathbb{M}$, then the functions $\mu,|\mu|$ and $\nu$ are all uniformly continuous on $E \cap \mathbb{M}$.

Proof. The uniform continuity of $\mu$ follows from the relation

$$
\begin{aligned}
|\mu(A)-\mu(B)| & =|\mu(A-B)-\mu(B-A)| \leq|\mu(A-B)|+|\mu(B-A)| \\
& \leq|\mu|(A-B)+|\mu(B-A)|=\rho(A, B)
\end{aligned}
$$

where $A, B \in E \cap \Re$.

Note that $\mu$ can be replaced by $|\mu|$ in the above relation which proves the desired result for $|\mu|$.

To prove the result for $\nu$, let $\epsilon>0$. Since $\nu \ll|\mu|$ on $E$, there exists a $\delta>0$ such that $|\nu(F)|<\epsilon / 2$ for all $F \in E \cap \pi$ such that $|\mu|(E)<\delta$. Then if $A, B \in E \cap M$, we have

$$
\begin{aligned}
|\nu(A)-\nu(B)| & =|\nu(A-B)-\nu(B-A)| \\
& \leq|\nu(A-B)|+|\nu(B-A)|<\epsilon / 2+\epsilon / 2=\epsilon
\end{aligned}
$$

when $\rho(A, B)=|\mu|(A-B)+|\mu|(B-A)<\delta$. Thus $\nu$ is uniformly continuous on $E \cap \pi$.

Lemma 2. Let $\bar{E} \in \mathbb{M}_{0}$. Then $\bar{E} \cap \mathbb{M}$ is a closed subset of $\mathbb{M}_{0}$.

Proof. Let $E_{n} \in \bar{E} \cap \mathbb{M}, F \in \mathbb{M}_{0}$ and $\rho\left(E_{n}, F\right) \rightarrow 0$ as $n \rightarrow \infty$. The inequality $|\mu|\left(F-E_{n}\right) \leq \rho\left(E_{n}, F\right)$ implies that $|\mu|\left(F-E_{n}\right) \rightarrow 0$ as $n \rightarrow \infty$. Since each $E_{n} \subseteq \bar{E}$, we have $F-\bar{E} \subseteq F-E_{n}$ for all $n$. Therefore, $|\mu|(F-\bar{E})$ $\leq|\mu|\left(F-E_{n}\right)$. Letting $n \rightarrow \infty$, we then obtain $|\mu|(F-\bar{E})=0$, i.e. $F \subseteq \bar{E}[\mu]$. Thus there exists a set $E \in \mathbb{M}_{0}$ such that $E \subseteq \bar{E}$ and $E=F[\mu]$. Since $E$ and $F$ are regarded identical in the metric space $\prod_{0}, \bar{E} \cap \pi$ is closed.

We now prove the following local existence theorem which is analogous to Carathéodory's existence theorem for ordinary differential equations. 
Theorem 1. Let $E_{0} \subset E_{1}$ where $E_{0}, E_{1} \in \mathbb{M}_{0}$ and suppose that $\left(E_{1}-E_{0}\right)$ $\cap \pi$ is compact in the topology generated by the metric $\rho$ defined by (2.1). Define

$$
\Lambda_{a}\left(E_{1}\right)=\left\{\lambda \in \Lambda\left(E_{1}\right): V_{E_{1}-E_{0}}(\lambda-\nu) \leq a\right\}
$$

where $V_{E_{1}}-E_{0}$ denotes the variation on $E_{1}-E_{0}$. Let $f$ satisfy the following

(i) $f\left(x, \lambda_{x}\right)$ is measurable on $E_{1}$ for each fixed $\lambda \in \Lambda_{a}\left(E_{1}\right)$;

(ii) if $\lim _{k \rightarrow \infty} \lambda^{k}(E)=\bar{\lambda}(E), E \in E_{1} \cap \mathbb{M}$, then $\lim _{k \rightarrow \infty} f\left(x, \lambda_{x}^{k}\right)=f\left(x, \bar{\lambda}_{x}\right)$ for each $x \in E_{1}$;

(iii) there exists a $\mu$-integrable function $U$ on $E_{1}$ such that $\left|f\left(x, \lambda_{x}\right)\right|$ $\leq U(x)$ for all $\lambda \in \Lambda_{a}\left(E_{1}\right)$.

Suppose further that there exists a "g-class" for $E_{1}$ such that given any two sets in $\mathcal{G}$, one is contained in the other. Let $\mu$ be nonatomic, and $\nu \ll|\mu|$ on $\left(E_{1}-E_{0}\right) \cap \Uparrow$. Then there exists a solution of (1.1) on some set $\bar{E}$ in $\Pi_{0}$. $\bar{E}$ and

Proof. Since $\mu$ is nonatomic, there exists a set $\bar{E} \in \mathcal{G}$ such that $E_{0} \subset$

$$
\int_{\bar{E}-E_{0}} U(x) d|\mu| \leq a .
$$

For each $j=1,2, \ldots$, there exist sets $E_{k}^{j} \in \bar{E} \cap \Theta(k=0,1, \ldots, j)$ such that

$$
E_{0}=E_{0}^{j} \subset E_{1}^{j} \subset E_{2}^{j} \subset \ldots \subset E_{j}^{j}=\bar{E}[\mu]
$$

and

$$
|\mu|\left(E_{k}^{j}-E_{k-1}^{j}\right)=(1 / j)|\mu|\left(\bar{E}-E_{0}\right), \quad k=1,2, \ldots, j .
$$

Thus, if $r_{j}=(1 / j)|\mu|\left(\bar{E}-E_{0}\right)$, then

$$
|\mu|\left(E_{k}^{j}\right)=|\mu|\left(E_{0}\right)+k r_{j}, \quad k=1,2, \ldots, j .
$$

For $E \in \bar{E} \cap 乌$, define

$$
\begin{array}{rlrl}
\lambda^{j}(E) & =\nu(E) & & \text { for } E \subseteq E_{1}^{j}, \\
& =\nu(E)+\int_{G-E_{0}} f\left(x, \lambda_{x}^{j}\right) d \mu & & \text { for } E_{k}^{j} \subset E \subseteq E_{k+1}^{j}, \\
& k=1,2, \ldots, j-1,
\end{array}
$$

where $G \in \bar{E} \cap \mathcal{G}$ is such that $E_{k-1}^{j} \subset G \subseteq E_{k}^{j}$ and

$$
|\mu|\left(G-E_{k-1}^{j}\right)=|\mu|\left(E-E_{k}^{j}\right), \quad k=1,2, \ldots, j-1 .
$$


Clearly $\lambda^{1}$ is defined on $\bar{E} \cap \oint$. For any fixed $j \geq 1$, the first formula in (2.5) defines $\lambda^{j}$ on $E_{1}^{1} \cap \mathcal{G}$ and the second formula extends $\lambda^{j}$ on $E_{2}^{j} \cap \mathcal{G}_{\text {. }}$ Also note that if $\lambda^{j}$ is defined on $E_{k}^{j} \cap \oint$ for $1<k<j$, then the second formula defines $\lambda^{j}$ on $E_{k+1}^{j} \cap \mathcal{G}$. Thus (2.5) defines $\lambda^{j}$ on $\bar{E} \cap \mathcal{G}$ which can then be extended to the $\sigma$-algebra $\bar{E} \cap M$. Furthermore, since any $\lambda \in \Lambda(E)$, where $E \subseteq E_{1}$, can be extended to a measure $\lambda_{1} \in \Lambda\left(E_{1}\right)$, by defining $\lambda_{1}(F)$ $=\lambda(E \cap F)$ for $F \in E_{1} \cap \mathbb{M}$, it follows by (iii) that $\left|f\left(x, \lambda_{x}^{j}\right)\right| \leq U(x)$. Therefore,

$$
V_{\bar{E}-E_{0}}\left(\lambda^{j}-\nu\right) \leq \int_{\bar{E}-E_{0}} U(x) d|\mu| \leq a .
$$

Thus, $\lambda^{j} \in \Lambda_{a}(\bar{E})$.

Let $E_{1}, E_{2} \in\left(\bar{E}-E_{0}\right) \cap \pi$. Then

$$
\begin{aligned}
\lambda^{j}\left(E_{1}\right)-\lambda^{j}\left(E_{2}\right)= & \nu\left(E_{1}-E_{2}\right)-\nu\left(E_{2}-E_{1}\right) \\
& +\int_{E_{1}-E_{2}} f\left(x, \lambda_{x}^{j}\right) d \mu-\int_{E_{2}-E_{1}} f\left(x, \lambda_{x}^{j}\right) d \mu .
\end{aligned}
$$

Therefore,

$$
\begin{aligned}
\mid \lambda^{j}\left(E_{1}\right) & -\lambda^{j}\left(E_{2}\right) \mid \\
& \leq|\nu|\left(\left(E_{1}-E_{2}\right) \cup\left(E_{2}-E_{1}\right)\right)+\int_{\left(E_{1}-E_{2}\right) \cup\left(E_{2}-E_{1}\right)} U(x) d|\mu| .
\end{aligned}
$$

Since $\nu \ll|\mu|$ on $\left(E_{1}-E_{0}\right) \cap \Uparrow$, by hypothesis, and $\omega \ll|\mu|$ where $\omega(E)=$ $\int_{E} U(x) d|\mu|, \nu$ and $\omega$ are uniformly continuous on $\left(\bar{E}-E_{0}\right) \cap \pi$ by Lemma 1 . It therefore follows from (2.7) that $\left\{\lambda^{j}\right\}$ is an equicontinuous set of functions on $\left(\bar{E}-E_{0}\right) \cap M$. Also, since $\lambda^{j} \in \Lambda_{a}(\bar{E}),\left\{\lambda^{j}\right\}$ is uniformly bounded on $\left(\bar{E}-E_{0}\right) \cap \Re$. By Lemma 2, $\left(\bar{E}-E_{0}\right) \cap \pi$ is a closed (and hence compact) subset of the compact space $\left(E_{1}-E_{0}\right) \cap M$. Therefore, by the $A_{\text {rzela-Ascoli }}$ theorem, $\left\{\lambda^{j}\right\}$ is conditionally compact in the space $C\left(\left(\bar{E}-E_{0}\right) \cap\right)$ of all bounded continuous scalar functions on $\left(\bar{E}-E_{0}\right) \cap$ M. Hence there exists a subsequence $\left\{\lambda^{j} n\right\}$ of $\left\{\lambda^{j}\right\}$ and a function $\bar{\lambda} \epsilon C\left(\left(\bar{E}-E_{0}\right) \cap M\right)$ such that $\lambda^{j} n$ $\rightarrow \bar{\lambda}$ uniformly on $\left(\bar{E}-E_{0}\right) \cap \mathbb{M}$ as $n \rightarrow \infty$. We extend $\bar{\lambda}$ to $\bar{E} \cap \mathbb{M}$ by defining $\bar{\lambda}(E)=\nu(E)$ for $E \in E_{0} \cap M$. Then we have

$$
\lim _{n \rightarrow \infty} \lambda^{j} n(E)=\bar{\lambda}(E), \quad E \in \bar{E} \cap \mathbb{M} \text {. }
$$

It now follows, by (ii), that

$$
\lim _{n \rightarrow \infty} f\left(x, \lambda_{x}^{j_{n}}\right)=f\left(x, \lambda_{x}\right), \quad x \in \bar{E}-E_{0} .
$$

By Lebesgue's dominated convergence theorem, we obtain 
(2.9) $\lim _{n \rightarrow \infty} \int_{E-E_{0}} f\left(x, \lambda_{x}^{j} n\right) d \mu=\int_{E-E_{0}} f\left(x, \lambda_{x}\right) d \mu, \quad E \in \bar{E} \cap \mathcal{G}$.

Further,

$$
\begin{aligned}
\left|\int_{E-G} f\left(x, \lambda_{x}^{j_{n}}\right) d \mu\right| & \leq \int_{E-G}\left|f\left(x, \lambda_{x}^{j_{n}}\right)\right| d|\mu| \\
& \leq \int_{E-G} U(x) d|\mu| \rightarrow 0 \text { as } n \rightarrow \infty,
\end{aligned}
$$

since, by (2.6) and (2.4),

Therefore,

$$
|\mu|(E-G)=|\mu|\left(E_{k}^{j_{n}}-E_{k-1}^{j_{n}}\right)=r_{j_{n}} \rightarrow 0 \text { as } n \rightarrow \infty .
$$

$$
\lim _{n \rightarrow \infty} \int_{E-G} f\left(x, \lambda_{x}^{j_{n}}\right) d \mu=0
$$

By (2.5), we have

$$
\begin{aligned}
\lambda^{j_{n}}(E)= & \nu(E) \quad \text { for } E \subseteq E_{1}^{j_{n}}, \\
= & \nu(E)+\int_{E-E_{0}} f\left(x, \lambda_{x}^{j_{n}}\right) d \mu \\
& -\int_{E-G} f\left(x, \lambda_{x}^{j_{n}}\right) d \mu \text { for } E_{k}^{j_{n}} \subset E \subseteq E_{k+1}^{j_{n}} .
\end{aligned}
$$

Taking limits as $n \rightarrow \infty$, we obtain, by using (2.8), (2.9) and (2.10),

$$
\bar{\lambda}(E)=\nu(E)+\int_{E-E_{0}} f\left(x, \bar{\lambda}_{x}\right) d \mu .
$$

Hence $\bar{\lambda}$ is a solution of (1.1) on $\bar{E}$. This completes the proof.

Remark. Let $E_{1}=\left[t_{0}, t_{1}\right], E_{0}=\varnothing, \mu$ be the Lebesgue measure and $\Theta_{=}$ $\left\{\left[t_{0}, t\right]: t \in\left[t_{0}, t_{1}\right]\right\}$. For each $s \in\left[t_{0} ; t_{1}\right]$, let $\lambda_{s}=\lambda\left[t_{0}, s\right]=x(s)$ and $\nu\left[t_{0}, s\right]=h(s)$. Then (1.1) takes the form

$$
x(t)=h(t)+\int_{t_{0}}^{t} f(s, x(s)) d s .
$$

In this case Theorem 1 reduces to Caratheodory's existence theorem for the integral equation (2.11).

3. Integral inequalities and extremal solutions. Throughout this section, we shall assume $\mu$ to be a positive measure so that $|\mu|=\mu$, and $\Lambda(E)$ will denote the set of real measures on $E \cap M$.

We first prove a fundamental integral inequality. 
Theorem 2. Let $\bar{E} \in \mathbb{N}$ with $\mu(\bar{E})<\infty$, and let $\nu, \lambda^{1}, \lambda^{2} \in \Lambda(E)$. Suppose that $\mu$ is nonatomic and $\lambda^{1}, \lambda^{2} \ll \mu$. Let $\lambda^{1}, \lambda^{2}$ satisfy the following conditions:

(i) if $\lambda^{1}(E) \leqq \lambda^{2}(E)$ for all $E \in \bar{E} \cap M$ satisfying $A \subset E \subset B$ then $f\left(x, \lambda_{x}^{1}\right) \leq f\left(x, \lambda_{x}^{2}\right)$ for all $x \in B-A$;

(ii) one of the inequalities

$$
\begin{aligned}
& \lambda^{1}(E) \leq \nu(E)+\int_{E-E_{0}} f\left(x, \lambda_{x}^{1}\right) d \mu \\
& \lambda^{2}(E) \leq \nu(E)+\int_{E-E_{0}} f\left(x, \lambda_{x}^{2}\right) d \mu
\end{aligned} \quad(E \in \bar{E} \cap \mathbb{N})
$$

is strict;

Then

(iii) $\lambda^{1}\left(E_{0}\right)<\lambda^{2}\left(E_{0}\right)$.

$$
\lambda^{1}(E)<\lambda^{2}(E) \text { whenever } E \in \bar{E} \cap \mathbb{M} \text { and } E_{0} \subseteq E[\mu] \text {. }
$$

Proof. Suppose that (3.2) is not true. Then the set $\mathcal{E}=\{E \in \bar{E} \cap \mathbb{M}$ : $\left.\lambda^{1}(E) \geq \lambda^{2}(E), E_{0} \subseteq E[\mu]\right\}$ is not empty. We shall first show that $\mathcal{E}_{\text {is }}$ closed.

Let $E_{n} \in \mathcal{E}^{0}$ and $\rho\left(E_{n}, F\right) \rightarrow 0$ as $n \rightarrow \infty$. By Lemma $1, \lambda^{1}$ and $\lambda^{2}$ are continuous on $\bar{E} \cap M$. Therefore,

$$
\lim _{n \rightarrow \infty} \lambda^{i}\left(E_{n}\right)=\lambda^{i}(F), \quad i=1,2 .
$$

Since $\lambda^{1}\left(E_{n}\right) \geq \lambda^{2}\left(E_{n}\right),(3.3)$ implies $\lambda^{1}(F) \geq \lambda^{2}(F)$. Also, since $E_{0} \subseteq E_{n}[\mu]$ we have $\mu\left(E_{0}-F\right) \leq \mu\left(E_{n}-F\right) \leq \rho\left(E_{n}, F\right)$. Letting $n \rightarrow \infty$, we obtain $\mu\left(E_{0}-F\right)=0$, i.e. $E_{0} \subseteq F[\mu]$. Thus $F \in \mathcal{E}$. Hence $\mathscr{E}$ is closed.

By (iii), it is clear that $E_{0} \notin \mathcal{E}$. Since $\mathcal{E}$ is closed, there exists an $E_{1} \in \mathcal{E}$ such that $0<\rho\left(E_{0}, E_{1}\right)=\inf _{E \in \mathcal{E}} \rho\left(E_{2}, E\right)$. Thus,

$$
\lambda^{1}(E)<\lambda^{2}(E), \quad E_{0} \subseteq E \subseteq E_{1}[\mu] .
$$

Since $\mu$ is nonatomic, there exists a sequence $A_{n} \in \bar{E} \cap \pi$ such that $E_{0} \subseteq$ $A_{n} \subset E_{1}$ and $\rho\left(A_{n}, E_{1}\right) \downarrow 0$. By (3.4), $\lambda^{1}\left(A_{n}\right)<\lambda^{2}\left(A_{n}\right)$. Taking limits as $n$ $\rightarrow \infty$, we obtain $\lambda^{1}\left(E_{1}\right) \leq \lambda^{2}\left(E_{1}\right)$. But $E_{1} \in E_{\text {. Therefore, }}$

$$
\lambda^{1}\left(E_{1}\right)=\lambda^{2}\left(E_{1}\right)
$$

It follows from (3.4), (3.5) and (i) that

$$
f\left(x, \lambda_{x}^{1}\right) \leq f\left(x, \lambda_{x}^{2}\right) \text { for each } x \in E_{1}-E_{0} .
$$

The inequalities (3.1) and (3.6) now yield $\lambda^{1}\left(E_{1}\right)<\lambda^{2}\left(E_{1}\right)$ which contradicts 
(3.5). Hence the set $\mathcal{E}$ is empty. This proves the theorem.

We shall now introduce the notion of maximal and minimal solutions.

Definition. A maximal solution of (1.1) on $\bar{E}$ is a solution $\lambda^{M}$ of (1.1) satisfying the inequality $\lambda(E) \leq \lambda^{M}(E), E \in \bar{E} \cap \pi$, where $\lambda$ is any solution of (1.1) on $\bar{E}$. A minimal solution $\lambda^{m}$ is similarly defined by reversing the preceding inequality.

The following theorem establishes the existence of maximal and minimal solutions under the hypotheses of Theorem 1.

Theorem 3. Let the hypotheses of Theorem 1 be satisfied. Suppose that if $\lambda^{1}, \lambda^{2} \in \Lambda\left(E_{1}\right)$ and $\lambda^{1}(E) \leq \lambda^{2}(E)$ for all $E \in E_{1} \cap \Re$ such that $E_{0} \subseteq E \subseteq$ $E_{1}$ then $f\left(x, \lambda_{x}^{1}\right) \leq f\left(x, \lambda_{x}^{2}\right)$ for all $x \in E_{1}-E_{0}$. Then there exist a maximal solution and a minimal solution of (1.1) on some $\bar{E} \in \mathbb{M}$.

Proof. Let $\left\{\epsilon_{j}\right\}$ be a decreasing sequence of numbers tending to zero as $n \rightarrow \infty$. For each $j=1,2, \ldots$, define

$$
\begin{aligned}
& \nu^{j}(E)=\nu(E)+\epsilon_{j} \quad \text { if } E_{0} \subseteq E \quad\left(E \in E_{1} \cap \mathbb{M}\right) . \\
& =\nu(E) \quad \text { otherwise }
\end{aligned}
$$

Consider the integral equation

$$
\lambda^{j}(E)=\nu^{j}(E)+\int_{E-E_{0}} f\left(x, \lambda_{x}^{j}\right) d \mu .
$$

Since $\nu=\nu^{j}$ on $\left(E_{1}-E_{0}\right) \cap \mathbb{M}$, it follows that $\nu^{j} \ll \mu$ on $\left(E_{1}-E_{0}\right) \cap M$. Also, the set $\Lambda_{a}\left(E_{1}\right)$ defined by (2.2) is unaltered if $\nu$ is replaced by any $\nu^{j}$. By Theorem 1 , there exists a solution $\lambda^{j}$ of (3.7) on some $\bar{E} \in E_{1} \cap$ ) for each $j=1,2, \ldots$. The only requirement for $\bar{E}$ is to satisfy (2.3). We may therefore choose a single $\bar{E}$ for the domain of definitions of all the solutions $\lambda^{j}$ 's. It can be proved as in the proof of Theorem 1 that there exists a subsequence $\left\{\lambda^{j} n\right\}$ and a function $\lambda^{M}$ on $\bar{E} \cap \pi$ such that

$$
\lim _{n \rightarrow \infty} \lambda^{j} n(E)=\lambda^{M}(E)
$$

and

$$
\lim _{n \rightarrow \infty} \int_{E-E_{0}} f\left(x, \lambda_{x}^{j_{n}}\right) d \mu=\int_{E-E_{0}} f\left(x, \lambda_{x}^{M}\right) d \mu, \quad E \in \bar{E} \cap \mathbb{M} .
$$

Replace $j$ by $j_{n}$ in (3.7) and take limits as $n \rightarrow \infty$. Since $\nu^{j} \rightarrow \nu$ as $n \rightarrow \infty$, we obtain

$$
\lambda^{M}(E)=\nu(E)+\int_{E-E_{0}} f\left(x, \lambda_{x}^{M}\right) d \mu, \quad E \in \bar{E} \cap \mathbb{M}
$$


which shows that $\lambda^{M}$ is a solution of $(1.1)$ on $\bar{E}$.

We shall now show that $\lambda^{M}$ is the desired maximal solution of $(1.1)$ on $\bar{E}$. Let $\lambda$ be any solution of $(1.1)$ on $\bar{E}$. Then

$$
\begin{aligned}
\lambda\left(E_{0}\right) & <\lambda^{j_{n}}\left(E_{0}\right), \\
\lambda^{j} n(E) & >\nu(E)+\int_{E-E_{0}} f\left(x, \lambda_{x}^{j}{ }^{n}\right) d \mu \quad(E \in \bar{E} \cap \mathbb{M}), \\
\lambda(E) & =\nu(E)+\int_{E-E_{0}} f\left(x, \lambda_{x}\right) d \mu .
\end{aligned}
$$

Theorem 2 then yields

$$
\lambda(E)<\lambda^{j_{n}}(E) \text { for } E_{0} \subseteq E \quad(E \in \bar{E} \cap \mathbb{M}) .
$$

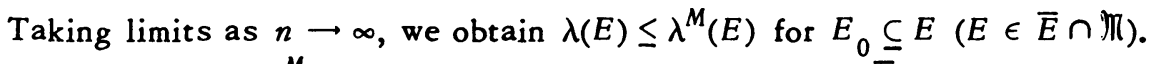
This shows that $\lambda^{M}$ is the maximal solution of (1.1) on $\bar{E}$.

The existence of minimal solution can similarly be proved.

Note. By applying Theroem 2, it can be shown that if $j<k$ (so that $\epsilon_{k}<$ $\epsilon_{j}$ ) then $\lambda^{k}(E)<\lambda^{j}(E)$ for all $E \in \bar{E} \cap \Re$. Hence the sequence $\lambda^{j}$ itself converges to $\lambda^{M}$ as $j \rightarrow \infty$. As such the question of choosing a convergent subsequence $\lambda^{j} n$ does not in fact arise in the above proof.

As another application of Theorem 2, we prove the following comparison theorem.

Theorem 4. Suppose that the assumptions of Theorem 3 are satisfied, and $\lambda^{M}$ is the maximal solution of (1.1) on $\bar{E}$. Let $\lambda^{*} \in \Lambda(\bar{E})$, and $\lambda^{*}(E)<\nu(E)$ $+\int_{E-E} f\left(x, \lambda_{x}^{*}\right) d \mu, E \in \bar{E} \cap M$. Then the inequality $\lambda^{*}\left(E_{0}\right) \leq \lambda^{M}\left(E_{0}\right)$ implies $\lambda^{*}(E) \leq \lambda^{M}(E), E \in \bar{E} \cap \mathbb{M}$.

Proof. Let $\lambda^{j}$ be any solution of (3.7). Since

$$
\lim _{j \rightarrow \infty} \lambda^{j}(E)=\lambda^{M}(E), \quad E \in \bar{E} \cap \mathbb{M}
$$

it is enough to show that

$$
\lambda^{*}(E)<\lambda^{j}(E), \quad E \in \bar{E} \cap \mathbb{M} .
$$

Observe that $\lambda^{*}\left(E_{0}\right)<\lambda^{j}\left(E_{0}\right)$ and $\lambda^{j}(E) \geq \nu(E)+\int_{E-E_{0}} f\left(x, \lambda_{x}^{j}\right) d \mu$. The inequality (3.8) now follows from Theorem 2. This completes the proof.

\section{REFERENCES}

1. P. C. Das, Nonlinear integral equations in a measure space, Proc. Amer. Math. Soc. 42 (1974), 181-185. 
2. R. R. Sharma, An abstract measure differential equation, Proc. Amer. Math. Soc. 32 (1972), 503-510. MR $45 \# 691$.

3. - Existence of solutions of abstract measure differential equations, Proc. Amer. Math. Soc. 35 (1972), 129-136. MR 46 \#3943.

4. - A measure differential inequality with applications, Proc. Amer. Math. Soc. 48 (1975), 87-97.

DEPARTMENT OF MATHEMATICS, REGIONAL INSTITUTE OF TECHNOLOGY, JAMSHEDPUR 831014, INDIA 